\title{
Deregulated Semantic Cognition Follows Prefrontal and Temporo-parietal Damage: Evidence from the Impact of Task Constraint on Nonverbal Object Use
}

\author{
Faye Corbett ${ }^{1}$, Elizabeth Jefferies ${ }^{2}$, and Matthew A. Lambon Ralph ${ }^{1}$
}

\begin{abstract}
Semantic cognition, which encompasses all conceptually based behavior, is dependent on the successful interaction of two key components: conceptual representations and regulatory control. Qualitatively distinct disorders of semantic knowledge follow damage to the different parts of this system. Previous studies have shown that patients with multimodal semantic impairment following CVA - a condition referred to as semantic aphasia (SA) - perform poorly on a range of conceptual tasks due to a failure of executive control following prefrontal and/or temporoparietal infarction [Jefferies, E., \& Lambon Ralph, M. A. Semantic impairment in stroke aphasia versus semantic dementia: A caseseries comparison. Brain, 129, 2132-2147, 2006]. Although a deficit of core semantic control would be expected to impair all modalities in parallel, most research exploring this condition has
\end{abstract}

\section{INTRODUCTION}

Semantic cognition incorporates the processes and representations that underlie our ability to comprehend the environment, including our understanding of words, pictures, environmental sounds, objects, and faces. It is, therefore, a key foundation for many everyday activities ranging from those in the verbal domain, such as holding a conversation, to more nonverbal tasks like using household objects (e.g., Bozeat, Lambon Ralph, Patterson, \& Hodges, 2002). Consequently, semantic deficits have a highly debilitating effect on patients' daily lives. Recent evidence suggests that semantic cognition is supported by two principal components that interact with one another: (1) a repository of amodal semantic representations and (2) executive control processes (Jefferies \& Lambon Ralph, 2006; Rogers et al., 2004). Within this system, amodal conceptual representations are supported by a "hub and spoke" framework in which information from modality-specific regions (the spokes) is drawn together in a central repository (the hub; Lambon Ralph \& Patterson, 2008; Patterson, Nestor, \& Rogers, 2007; Rogers et al., 2004; Lambon Ralph, McClelland, Patterson, Galton, \& Hodges, 2001). The resultant set of modality-invariant representations supports semantic-based generalizations

${ }^{1}$ University of Manchester, UK, ${ }^{2}$ University of York, UK focused on tasks in the verbal domain. In a novel exploration of semantic control in the nonverbal domain, therefore, we assessed eight patients with SA on two experiments that examined object use knowledge under different levels of task constraint. Patients exhibited three key characteristics of semantic deregulation: (a) difficulty using conceptual knowledge flexibly to support the noncanonical uses of everyday objects; (b) poor inhibition of semantically related distractor items; and (c) improved object use with the provision of more tightly constraining task conditions following verbal and pictorial cues. Our findings are consistent with the notion that a neural network incorporating the left inferior prefrontal and temporo-parietal areas (damaged in SA) underpins regulation of semantic activation across both verbal and nonverbal modalities.

of knowledge across concepts that might not share the same surface characteristics (Lambon Ralph, Sage, Jones, \& Mayberry, 2009). Through interaction with the executive control component, just the relevant aspect of knowledge within the rich repository can be brought to the fore.

Damage to the different components of semantic cognition leads to qualitatively distinct semantic disorders (Jefferies \& Lambon Ralph, 2006). Semantic dementia (SD) is a neurodegenerative disorder characterized by a selective semantic impairment in the context of relatively circumscribed atrophy of the anterior temporal lobe bilaterally (ATL). Patients exhibit a highly consistent impairment across a full range of modalities, suggesting that the ATL underpins a repository of amodal representations that gradually degrade in this condition (Binney, Embleton, Jefferies, Parker, \& Lambon Ralph, 2010; Nestor, Fryer, \& Hodges, 2006; Rogers et al., 2004; Mummery et al., 2000). Multimodal semantic impairments are also exhibited by some patients following a stroke; a pattern we have come to refer to as "semantic aphasia" (SA). Unlike SD, however, SA follows damage to left prefrontal and/or temporoparietal regions. Conceptual knowledge appears to remain largely intact in SA but a failure of regulatory control processes causes semantic cognition to become deregulated (Noonan, Jefferies, Corbett, \& Lambon Ralph, 2010; Jefferies \& Lambon Ralph, 2006). 
Most previous studies of semantic cognition have concentrated on the representational structure of conceptual knowledge (Rogers et al., 2004; Caramazza \& Shelton, 1998; Warrington \& Shallice, 1984). Comparatively less is known about the processes that support the application of semantic knowledge in specific situations or tasks (Koenig \& Grossman, 2007; Jefferies \& Lambon Ralph, 2006). Although many fMRI studies have examined aspects of semantic control in healthy participants (e.g., ThompsonSchill, 2003; Thompson-Schill, D'Esposito, Aguirre, \& Farah, 1997), there is only limited convergent evidence from neuropsychological populations (Thompson-Schill et al., 1998); a shortfall this study will help to address. Control is a critical feature of semantic processing because we know so many things about each concept and not all of this information will be useful in every situation. It is essential, therefore, to focus on the particular aspects of knowledge that are relevant in a given task, time, or context. Taking the Pyramids and Palm trees semantic association task as an example (Howard \& Patterson, 1992), in order to make the correct pairing between probe and target in each trial, the participant must converge on just the relevant relationship between the two items (e.g., pyramid and palm tree $\rightarrow$ "found in a desert") and reject other possible, but erroneous, associations (e.g., color and shape). The same control demands are true of this task regardless of whether concepts are tapped through word or picture stimuli. Indeed, control is crucial to all activities that draw on conceptual knowledge irrespective of modality, including nonverbal tasks such as object use (Corbett, Jefferies, Ehsan, \& Lambon Ralph, 2009). Moreover, semantic control needs to be exerted over time, even for the same object within a single task, because the critical aspects of knowledge can change over this period. Imagine, for instance, the many different applications of a knife while making a cheese and chutney sandwich, during which the same utensil could be used to open the packet, spread the butter, cut the bread, slice the cheese, scoop the pickle, and so forth. Different features of the knife must be brought to the fore one-by-one during the task, including multiple ways of holding and manipulating the object, while its canonical function (i.e., cutting) is most often inhibited in favor of more subordinate uses and even functions that are more closely associated with a different object (e.g., scooping $=$ spoon; Noonan et al., 2010).

Some recent studies have explored the consequences of semantic deregulation in the verbal domain (Noonan et al., 2010; Jefferies, Patterson, \& Lambon Ralph, 2008; Jefferies \& Lambon Ralph, 2006). SA patients' performance on semantic tasks was found to be dependent on the particular demands they placed on control processes. Straightforward picture naming requires a degree of control in order to select the correct verbal label from a number of competing responses. SA patients made frequent semantically driven mistakes during picture naming including associative errors (e.g., squirrel $\rightarrow$ "nuts"), which were indicative of their deregulated system. In line with this hypothesis, the performance of these patients is sensitive to cueing and con- straints provided by the examiner. For example, naming improved substantially when the target picture was preceded by a phonemic cue. In contrast, accuracy was diminished when the first phoneme of a semantic competitor was provided (Jefferies et al., 2008; Jefferies \& Lambon Ralph, 2006). Similar findings were observed in receptive tasks. When making semantic associations, for example, SA patients found it more difficult to match a homonymous probe concept (e.g., "FIRE") with a subordinate associate (e.g., "RIFLE") compared to a more dominant meaning (e.g., "нот"). Performance on the subordinate matching condition improved significantly, however, when the less dominant meaning of the probe item was cued by a preceding sentence (e.g., "Fire at will"; Noonan et al., 2010). These findings indicated that damage to the network underpinning control led to deregulation of normal semantic processing in SA. Consistent with this view, neuroimaging studies have commonly reported stronger activation of left prefrontal and/or temporo-parietal areas (damaged in SA) when healthy participants engage in semantic tasks requiring a greater degree of control (Badre, Poldrack, PareBlagoev, Insler, \& Wagner, 2005; Wagner, Pare-Blagoev, Clark, \& Poldrack, 2001; Thompson-Schill et al., 1997; Demb et al., 1995). For example, Rodd, Davis, and Johnsrude (2005) asked fMRI participants to judge whether a word was related to a preceding sentence. They found greater frontal and posterior temporal-parietal activation when the prime sentence contained words that were ambiguous due to their many competing meanings (e.g., "the shell was fired toward the tank" $\rightarrow$ "battle?"). Within this literature, there has been further debate about the nature of the semantic control mechanism, with some favoring a competition resolution function, others arguing for a role in controlled retrieval and, more recently, the suggestion that both processes are supported separately within the inferior frontal gyrus (Badre et al., 2005; Wagner et al., 2001; Thompson-Schill, D’Esposito, \& Kan, 1999; Thompson-Schill et al., 1997, 1998). Although this debate is both interesting and important, the present study was not designed to differentiate between these views but rather to explore the role of cognitive control more generally in nonverbal semantic cognition, as described below.

Two previously separate literatures have reached convergent conclusions about semantic processing. fMRI studies with healthy control participants have highlighted the role of a PFC-temporo-parietal semantic control network (Thompson-Schill, 2003; Wagner et al., 2001; ThompsonSchill et al., 1997; Demb et al., 1995), whereas patients with semantic aphasia have been found to exhibit multimodal semantic impairment following damage to the same regions (Jefferies \& Lambon Ralph, 2006). The latter body of work suggests that the semantic impairment exhibited by SA patients is attributable to damage to the control component of semantic cognition (Noonan et al., 2010; Corbett et al., 2009). If the semantic impairment exhibited by SA patients is a domain-general problem, patients should exhibit parallel deficits in the nonverbal domains as well. In both the 
imaging and neuropsychological literature, however, the nature of the semantic control function has only really been explored using verbal materials. One previous study utilized the rich and demanding domain of object use to assess the SA patients' nonverbal semantic control. The results indicated that their object use deficit was consistent with a failure of regulatory control processes, although this study did not experimentally vary the control demands of the object use tasks directly (Corbett et al., 2009). In the present study, therefore, we designed two experiments to examine the effect of changing task constraint on object use knowledge. These were used to assess a group of eight SA patients. Specifically, three aspects of control were targeted that have previously been shown to affect verbal semantic processing in this patient group (e.g., Noonan et al., 2010; Jefferies, Baker, Doran, \& Lambon Ralph, 2007; Jefferies \& Lambon Ralph, 2006): (1) the ability to focus on nondominant aspects of knowledge; (2) the effect of closely related, and therefore highly distracting foil responses; and (3) the impact of bolstering central aspects of a concept with increasing task constraint (e.g., picture and word based cues) such that participants are more readily able to make use of the relevant aspect of their knowledge. Assuming that our predictions are supported, not only will this study be consistent with the hypothesis that a failure of top-down regulation leads to multimodal semantic impairments in this patient group, it will also provide further convergence between neuropsychology and neuroimaging studies, which have implicated left prefrontal and temporo-parietal regions (damaged in SA) in the regulation of a wide variety of semantic tasks across modalities.

\section{METHODS}

\section{Patients}

Eight patients with aphasia following a stroke were recruited from speech and language therapy services and stroke clubs in Manchester, U.K. Patients were recruited to the study if they obtained scores outside the normal range on both word and picture versions of the Camel and Cactus semantic association task (Bozeat, Lambon Ralph, Patterson, Garrard, \& Hodges, 2000). All of the patients had chronic impairment resulting from a stroke that occurred at least a year before the study (see Table 1 for further background information).

\section{Lesion Analyses}

CT/MRI scans were available for seven out of eight patients. A previous CT scan was not available for P. G. but an associated radiological report described a left frontal lesion. No explicit reference was made to posterior damage in this case and contraindications prevented additional MR scanning. For the remaining seven patients, scans were manually traced onto Damasio's standardized templates (Damasio \& Damasio, 1989; 6/7 cases previously reported by Noonan et al., 2010). Analysis revealed damage affecting the left prefrontal region (particularly in BA 44) and/or temporo-parietal areas; most significantly BA 37 of posterior temporal cortex and white matter areas of inferior parietal cortex (BA 39/40). Frontal as well as temporo-parietal sites were damaged in four out of seven cases, whereas three out of seven patients had damage restricted to the posterior regions (see Table 1 for a breakdown of patients' lesions).

\section{Control Participants}

The SA patients were compared with a group of eight ageand education-matched control participants [mean age: SA group $=64$ years, control group $=70$ years, $t(14)>1$; mean years in education: SA group $=10.5$, control group $=$ $11.5, t(14)=1.54, n s]$. All control participants scored within the normal range on the Mini-Mental State Examination (Folstein, Folstein, \& McHugh, 1976).

\section{General Neuropsychological Examinations}

The patients were examined on a range of general neuropsychological tasks, including: forward and backward-digit span (Wechsler, 1987), nonword and real-word repetition (PALPA tests 8 and 9; Kay, Lesser, \& Coltheart, 1992), the Cookie theft fluency assessment from the Boston Diagnostic Aphasia Examination (BDAE; Goodglass, 1983), and the Visual Object Space Perception Battery (Warrington \& James, 1991). Attentional and executive skills were assessed with the following tests: the Wisconsin Card Sorting Task (WCST; Stuss et al., 2000; Milner, 1964), the Elevator Counting subtests of the Test of Everyday Attention (with and without distraction; Robertson, Ward, Ridgeway, \& Nimmo-Smith, 1994), the Brixton Spatial Rule Attainment Task (Burgess \& Shallice, 1996), and the Coloured Progressive Matrices Test of Nonverbal Reasoning (Raven, 1962).

All patients scored within the impaired range on multiple attentional/executive assessments except the very mildest case (H. N.), who nonetheless scored below the normal range on the Brixton Spatial Rule Attainment Task and had a reduced forward digit span (see Table 2).

\section{Background Semantic Memory Assessments}

A battery of tasks was used to assess knowledge of 64 items drawn from six categories, including animals, birds, fruit, household items, tools, and vehicles. The tasks were:

(i) The Camel and Cactus Test (CCT; Bozeat et al., 2000). In this task, participants were required to select which of four response options was most closely related to a probe item. For example, when the probe item was "camel," participants had to select "cactus" as the correct associate and reject the other possible response options (tree, sunflower, rose). The test was administered in both word and picture formats. 
Table 1. Background Details and Lesion Characteristics for SA Patients

\begin{tabular}{|c|c|c|c|c|c|c|c|c|c|c|c|c|c|c|c|c|c|c|c|c|}
\hline \multirow[b]{2}{*}{ Case } & \multirow[b]{2}{*}{ Age } & \multirow[b]{2}{*}{ Sex } & \multirow{2}{*}{$\begin{array}{c}\text { Education } \\
\text { (Leaving } \\
\text { Age) }\end{array}$} & \multirow[b]{2}{*}{ Aphasia Type } & \multirow[b]{2}{*}{ Etiology of Stroke } & \multirow[b]{2}{*}{$\begin{array}{l}\text { L Frontal } \\
\text { Lesion }\end{array}$} & \multirow{2}{*}{$\begin{array}{c}\text { L Temporo- } \\
\text { parietal } \\
\text { Lesion }\end{array}$} & \multicolumn{2}{|c|}{$D L P F C$} & \multirow{2}{*}{$\frac{o r b I F C}{B A 47}$} & \multirow{2}{*}{$\frac{\operatorname{trIFG}}{B A 45}$} & \multirow{2}{*}{$\frac{o p I F G}{B A 44}$} & \multirow{2}{*}{$\frac{S T G}{B A 22}$} & \multirow{2}{*}{$\frac{M T G}{B A 21}$} & \multirow{2}{*}{$\frac{I T G}{B A 20}$} & \multirow{2}{*}{$\frac{F G}{B A 36}$} & \multirow{2}{*}{$\frac{P O T}{B A 37}$} & \multirow{2}{*}{$\frac{A G}{B A 39}$} & \multirow{2}{*}{$\frac{S M G}{B A 40}$} & \multirow{2}{*}{$\frac{T P}{B A 38}$} \\
\hline & & & & & & & & $B A 9$ & $B A 46$ & & & & & & & & & & & \\
\hline H. N. & 78 & M & 14 & Anomic/TSA & Ischemic & $\mathrm{x}$ & $\mathscr{V}$ & - & - & - & - & - & - & 2 & 1 & - & 2 & $\mathrm{~W}$ & - & - \\
\hline S. C. & 76 & M & 16 & Anomic/TSA & Hemorrhage & $\mathrm{x}$ & $\swarrow$ & - & - & - & - & - & - & - & 2 & - & 2 & 2 & $\mathrm{~W}$ & - \\
\hline P. G. & 59 & M & 18 & TSA & $\begin{array}{c}\text { Subarachnoid } \\
\text { hemorrhage }\end{array}$ & $\swarrow$ & a & \multicolumn{13}{|c|}{ No MRI available } \\
\hline N. Y. & 63 & M & 15 & Conduction & Not known & 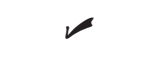 & $\prec$ & 1 & 1 & 2 & 2 & 2 & 1 & - & - & - & - & 2 & 2 & - \\
\hline B. B. ${ }^{b}$ & 55 & $\mathrm{~F}$ & 16 & $\begin{array}{l}\text { Mixed } \\
\text { transcortical }\end{array}$ & $\begin{array}{c}\text { Subarachnoid } \\
\text { hemorrhage }\end{array}$ & $\prec$ & $\prec$ & - & - & 2 & 2 & 2 & 2 & - & - & - & - & - & - & - \\
\hline K. A. & 74 & M & 14 & Global & $\begin{array}{l}\text { Thromboembolic/ } \\
\text { partial hemorrhage }\end{array}$ & $\swarrow$ & $\swarrow$ & - & - & - & - & 2 & 2 & 1 & - & - & 2 & - & 2 & - \\
\hline M. E. & 36 & $\mathrm{~F}$ & 16 & TSA & $\begin{array}{c}\text { Subarachnoid } \\
\text { hemorrhage }\end{array}$ & $\mathrm{x}$ & $\swarrow$ & - & - & - & - & - & - & 2 & 2 & 2 & 2 & $\mathrm{w}$ & $\mathrm{w}$ & - \\
\hline L. S. & 71 & M & 15 & TSA & Not known & $\swarrow$ & $\swarrow$ & 2 & 1 & 1 & 2 & 2 & - & 2 & 2 & - & 2 & 2 & 1 & - \\
\hline \multicolumn{8}{|c|}{$\%$ patients with gray matter damage } & 29 & 29 & 43 & 43 & 57 & 43 & 57 & 57 & 14 & 71 & 43 & 43 & 0 \\
\hline \multicolumn{8}{|c|}{$\%$ patients with gray or white matter damage } & 29 & 29 & 43 & 43 & 57 & 43 & 57 & 57 & 14 & 71 & 71 & 71 & 0 \\
\hline
\end{tabular}

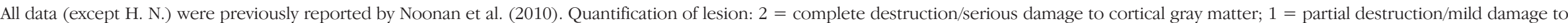

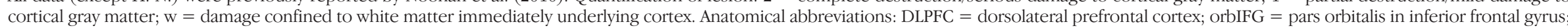

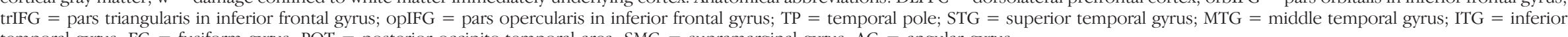
temporal gyrus; FG = fusiform gyrus; POT = posterior occipito-temporal area; SMG = supramarginal gyrus; $\mathrm{AG}=$ angular gyrus .

${ }^{\mathrm{a}}$ Not mentioned on scan report.

${ }^{b}$ B. B. showed additional signs of ventricular enlargement in the left hemisphere. 
Table 2. Background Neuropsychological Assessments

\begin{tabular}{|c|c|c|c|c|c|c|c|c|c|c|c|}
\hline Task & $\operatorname{Max}$ & $\begin{array}{l}\text { Control Mean } \\
\text { (SD) }\end{array}$ & $\begin{array}{c}\text { SA } \\
\text { Average }\end{array}$ & $H . N$. & S. $C$. & P. G. & $N . Y$. & B. B. & K. A. & M. E. & L. $S$. \\
\hline \multicolumn{12}{|l|}{ Repetition } \\
\hline PALPA 8 nonword repetition total & 30 & & 22 & 11 & 26 & 22 & 12 & 25 & NT & 28 & 27 \\
\hline PALPA 9 real word repetition total & 80 & $73^{\mathrm{a}}$ & 74 & $69^{\mathrm{b}}$ & 78 & 73 & $65^{\mathrm{b}}$ & 77 & NT & 80 & 77 \\
\hline \multicolumn{12}{|l|}{ Comprebension } \\
\hline Naming & 64 & $62.3(1.6)$ & 25 & $50^{\mathrm{b}}$ & $28^{\mathrm{b}}$ & $46^{\mathrm{b}}$ & $55^{\mathrm{b}}$ & $10^{\mathrm{b}}$ & $0^{\mathrm{b}}$ & $5^{\mathrm{b}}$ & $5^{\mathrm{b}}$ \\
\hline Word-picture & 64 & $63.7(.5)$ & 49 & $50^{\mathrm{b}}$ & $59^{\mathrm{b}}$ & $58^{\mathrm{b}}$ & $60^{\mathrm{b}}$ & $54^{\mathrm{b}}$ & $26^{\mathrm{b}}$ & $50^{\mathrm{b}}$ & $37^{\mathrm{b}}$ \\
\hline Picture CCT & 64 & $58.9(3.1)$ & 37 & 54 & $46^{\mathrm{b}}$ & $44^{\mathrm{b}}$ & $36^{\mathrm{b}}$ & $38^{\mathrm{b}}$ & $46^{\mathrm{b}}$ & $13^{\mathrm{b}}$ & $16^{\mathrm{b}}$ \\
\hline Word CCT & 64 & $60.7(2.06)$ & 38 & $54^{\mathrm{b}}$ & 56 & $40^{\mathrm{b}}$ & $39^{\mathrm{b}}$ & $30^{\mathrm{b}}$ & $36^{\mathrm{b}}$ & $34^{\mathrm{b}}$ & $16^{\mathrm{b}}$ \\
\hline \multicolumn{12}{|l|}{ Fluency } \\
\hline Category fluency (6) & - & $95.7(16.5)$ & 21 & $52^{\mathrm{b}}$ & $17^{\mathrm{b}}$ & $4^{\mathrm{b}}$ & $25^{\mathrm{b}}$ & $13^{\mathrm{b}}$ & NT & $25^{\mathrm{b}}$ & $11^{\mathrm{b}}$ \\
\hline Letter fluency (F, A, S) & - & $44.2(11.2)$ & 9 & $19^{\mathrm{b}}$ & 24 & $2^{\mathrm{b}}$ & $5^{\mathrm{b}}$ & $0^{\mathrm{b}}$ & $0^{\mathrm{b}}$ & $14^{\mathrm{b}}$ & $8^{\mathrm{b}}$ \\
\hline Cookie theft (words/minute) & - & & 46 & 85 & 84 & 27 & 1 & NT & 34 & 63 & 30 \\
\hline \multicolumn{12}{|l|}{ Attentional and Executive Tasks } \\
\hline TEA: counting without distraction & 7 & $6^{\mathrm{a}}$ & 5 & 7 & 7 & $3^{\mathrm{b}}$ & $3^{\mathrm{b}}$ & $4^{\mathrm{b}}$ & NT & 7 & $3^{\mathrm{b}}$ \\
\hline TEA: counting with distraction & 10 & $3^{\mathrm{a}}$ & 3 & 9 & $1^{b}$ & $0^{\mathrm{b}}$ & $2^{\mathrm{b}}$ & $0^{\mathrm{b}}$ & NT & 9 & $2^{\mathrm{b}}$ \\
\hline Raven's colored matrices (percentiles) & - & - & - & 50 & 50 & 50 & 50 & 50 & $5^{b}$ & $<5^{\mathrm{b}}$ & 10 \\
\hline WCST (number of categories) & 6 & $1^{\mathrm{a}, \mathrm{c}}$ & 2 & 6 & 6 & $0^{\mathrm{b}}$ & 2 & 1 & 1 & $0^{\mathrm{b}}$ & $0^{\mathrm{b}}$ \\
\hline Brixton spatial anticipation (correct) & 54 & $28^{\mathrm{a}}$ & 21 & $26^{\mathrm{b}}$ & $25^{\mathrm{b}}$ & $26^{\mathrm{b}}$ & 34 & $23^{\mathrm{b}}$ & $6^{\mathrm{b}}$ & $11^{\mathrm{b}}$ & $14^{\mathrm{b}}$ \\
\hline Digit span: forward & - & $5^{\mathrm{a}}$ & 4 & $4^{\mathrm{b}}$ & 6 & 6 & $3^{\mathrm{b}}$ & 5 & $0^{\mathrm{b}}$ & 6 & $4^{b}$ \\
\hline Digit span: backward & - & $2^{\mathrm{a}}$ & 2 & 3 & 2 & 2 & 2 & $0^{\mathrm{b}}$ & NT & 3 & $1^{\mathrm{b}}$ \\
\hline \multicolumn{12}{|l|}{ Visuospatial Tasks } \\
\hline VOSP dot counting & 10 & $8^{\mathrm{a}}$ & 7 & 8 & 10 & $5^{b}$ & 10 & 10 & $0^{\mathrm{b}}$ & $3^{\mathrm{b}}$ & $6^{\mathrm{b}}$ \\
\hline VOSP position discrimination & 20 & $18^{\mathrm{a}}$ & 17 & 19 & $17^{\mathrm{b}}$ & 20 & 20 & 18 & $14^{\mathrm{b}}$ & $15^{\mathrm{b}}$ & $16^{\mathrm{b}}$ \\
\hline VOSP number location & 10 & $7^{\mathrm{a}}$ & 8 & 9 & 10 & 9 & 10 & 8 & $6^{\mathrm{b}}$ & $2^{\mathrm{b}}$ & 8 \\
\hline VOSP cube analysis & 10 & $6^{\mathrm{a}}$ & 5 & $4^{\mathrm{b}}$ & 9 & 10 & $5^{b}$ & $2^{\mathrm{b}}$ & NT & $4^{\mathrm{b}}$ & $4^{\mathrm{b}}$ \\
\hline
\end{tabular}

Patients are arranged in order of their composite semantic scores generated from the four tasks in the 64-item semantic battery (H. N. obtained the highest score). NT $=$ not tested.

${ }^{a}$ Normal cutoff.

${ }^{\mathrm{b}}$ Denotes impaired scores (less than two standard deviations below mean).

${ }^{\mathrm{c}}$ Cutoff for $50-74$ year olds (regardless of educational level).

(ii) Spoken word-to-picture matching. For this task, participants selected a picture that corresponded to a verbal label given by the experimenter. An array of 10 pictures, all drawn from the same semantic category, was presented on each trial.

(iii) Picture naming. Participants were presented with a black and white line drawing of each item in the battery and asked to produce its name out loud.
Fluency was assessed for all six semantic categories assessed in the 64-item battery. Participants were given 1 min to produce as many exemplars as possible from each category. This was compared with verbal fluency for three letters ( $\mathrm{F}, \mathrm{A}, \mathrm{S})$.

All patients exhibited pronounced semantic impairments (see Table 2). Six of the eight patients scored within the impaired range (i.e., two standard deviations below the mean) 
on all four tasks in the 64-item semantic battery. H. N. and S. C. (the two mildest cases) obtained impaired scores on three out of four of these tasks. A single composite semantic score reflecting patients' overall level of impairment was generated from scores on all tasks in the 64-item battery. Patients are arranged by their composite semantic score (in descending order) in all tables and figures. Impaired performance on category and letter fluency tasks was exhibited by all patients.

\section{General Praxis}

The ability to imitate 10 meaningless gestures was used as a measure of general praxis (Goldenberg, 1996). Two points were awarded if the gesture was correct on the first attempt and a single point was awarded if the gesture was produced correctly on a second attempt. Imitation of meaningless gestures was performed with 85\% accuracy or greater by all participants except K. A. and L. S., whose vague movements achieved impaired scores of 30\% and $10 \%$ respectively.

\section{EXPERIMENT 1: CANONICITY AND DISTRACTION IN OBJECT SELECTION}

This experiment assessed patients' ability to use their understanding of object properties in a flexible way. We developed a conceptual judgment task in which participants were required to select an object that could be used to complete an everyday task (e.g., "kill a fly"). The test manipulated the ease with which a suitable object could be selected to complete the task. In half of the trials the canonical object (i.e., the object normally used for completing a task) was present in the array of response options (e.g., "kill a fly" $\rightarrow$ FLY SWAT). This condition was relatively easy to complete because the task and target object were strongly associated with one another in everyday life. In the remaining trials, the canonical object was not present but a plausible, noncanonical alternative constituted the correct response (e.g., "kill a fly" $\rightarrow$ Magazine). The noncanonical condition was more difficult because it required the participant to disregard knowledge about an object's normal purpose in order to determine if its properties rendered it suitable for completing the probe task. The strong predisposed use of the target object in the noncanonical condition could be driven by two factors: (1) existing functional and semantic knowledge about that object, and (2) highly rehearsed object affordances associated with the object (Pavese \& Buxbaum, 2002; Grèzes \& Decety, 2001; Gibson, 1979). In both cases, the cognitive control system has to inhibit the most familiar sources of information about the object's use and augment alternative elements of knowledge (e.g., object properties) that permit an alternative use of that object.

In the process of selecting the correct object for the task, it was also necessary to reject the distractor items pre- sented alongside the target response. All of the distractor items were unsuitable for the probe task, but some were easier to eliminate than others. In half of the trials, it was relatively straightforward to reject the foil items because not only were they unsuitable for the probe task, they were also unrelated to the canonical item (e.g., FLY SWAT, LIPSTICK). The distractors were more difficult to discount in the remaining trials because they shared a semantic relationship with the canonical item and would, therefore, become more strongly activated by the probe task than unrelated alternatives. For the task "kill a fly," related distractors included a MOUSE TRAP and FISHING NET, which share common general functions with the swat but could not be used in substitution in order to fulfil the target purpose.

\section{Methods}

Participants were presented with an array of six color photographs of different household objects. They were instructed to point to the object that would be most useful for performing an everyday probe task (e.g., "kill a fly"). A picture of the task recipient, as well as a short written description of the probe task, was supplied for the participant (e.g., "kill a fly" + picture of a fly). The description of the probe task was also read aloud by the experimenter. A total of 37 different everyday tasks were assessed under four conditions with a $2 \times 2$ design: (1) canonical object with unrelated distractors, (2) canonical object with semantically related distractors, (3) noncanonical object with unrelated distractors, and (4) noncanonical object with semantically related distractors. In the highly distracting condition, three of the five foils were semantically related and the remaining two foils were unrelated objects. The distinction between related and unrelated distractors was marked; while the related distractors came from the same semantic category as the canonical object and served closely related functions (e.g., shovel/rake), the unrelated distractors had no functional or categorical relationship with the target. The distractors were identical across the canonical/noncanonical conditions so that any difference across these conditions could be attributed to changes in the target rather than the foils. All 37 trials were assessed in each of the conditions in separate testing sessions. The four conditions were interleaved in a pseudorandom order. Pilot data from a student sample showed ceiling performance on all conditions of this task except the most difficult one. Based on this finding, age-matched control participants were examined on just the two most difficult conditions of the task (noncanonical selection with related and unrelated distractors) and showed a similar pattern of performance. Although these participants were not examined on the two easiest task conditions, it is highly probable that they would have achieved ceiling level scores due to the straightforward nature of the task in which everyday objects must be matched with their recipients. 


\section{Results}

In a repeated measures ANOVA, the patients showed significant main effects of canonicity [canonical item $>$ noncanonical item; $F(1,7)=107.43, p<.0001$; see Figure 1$]$ and semantic relationship of distractors [unrelated distractors $>$ related distractors; $F(1,7)=118.24, p<.0001]$. There was no interaction between the two factors $[F(1,7)<1]$.

The control participants were assessed on the two most difficult conditions of this task on which they attained ceiling level scores (noncanonical object selection: with unrelated distractors $97 \%$ accuracy; with related distractors 91\%; see Figure 1). When the control group's performance was compared with that of the SA group, there were significant main effects of distractor relationship [unrelated > related: $F(1,14)=30.44, p<.001]$ and group [controls $>$ SA patients: $F(1,14)=82.89, p<.0001$ ], and a significant interaction between these two factors $[F(1$, $14)=5.02, p=.042]$. In Bonferroni-adjusted planned comparisons, the SA patients scored more poorly than the control participants in both conditions [noncanonical selection: with unrelated distractors $t(14)=7.01, p<$ .0002 ; with related distractors $t(14)=10.83, p<.0002]$.

\section{EXPERIMENT 2: BOLSTERING OBJECT USE WITH TASK CONSTRAINT}

Assuming that a core control component has been damaged in the context of semantic aphasia, then expressive object use tasks should be susceptible to the effects of changing constraint in line with the results for the receptive task reported above. In our second experiment, we used four tasks to assess object use demonstration under different levels of task constraint.

\section{Methods}

We assessed the patients' ability to demonstrate the normal use of 36 household items (kitchen utensils/stationery/ tools) under four levels of constraint in a $2 \times 2$ design. In the least constrained condition, participants were asked to mime the normal use of the objects in response to their verbal label (e.g., "I would like you to show me how you would use a hammer"). The next task on the continuum offered a little more constraint as, in addition to the verbal prompt, participants were shown a picture of the object they were asked to mime. In the third condition, the task was made easier again by providing the participant with the actual object to demonstrate with. In the final and most constrained condition, participants demonstrated object use with the object in hand (e.g., hammer) but also with a picture of its recipient (e.g., a nail partly hammered into wood) and full verbal instructions (e.g., "I would like you to show me how you would use the hammer to bash a nail into wood"). In summary, the four conditions were: (1) mime no cue; (2) mime with picture cue (of object); (3) object use with no cue; and (4) object use with picture cue (picture of recipient provided). It was anticipated that each of these conditions would be more constraining than the previous one. A verbal cue was provided in addition to a picture cue in the fourth condition so that object use could be examined under the most constrained conditions possible.

The demonstrations were videotaped and later scored for accuracy against a predetermined set of target features derived from control participants' object demonstrations, a method shown previously to have high interrater agreement (Bozeat et al., 2002). Object use scores comprised three components: hold (including grasp and position on the object), movement, and orientation. Points were lost from the total accuracy score if an essential feature of the object's use was omitted from a demonstration. There were a small number of instances in which a participant clearly indicated how an object would be used but could not demonstrate its action due to hemiplegia. For example, a participant might pick up the hand drill and demonstrate how it would be held at the top of the object but then place the drill onto the table into order to demonstrate how the second handle would be turned. Points were not deducted
Figure 1. Canonicity and distraction in object selection. Control participants assessed on the two most difficult conditions only (noncanonical target + related/unrelated distractors). Error bars show standard error of the mean.

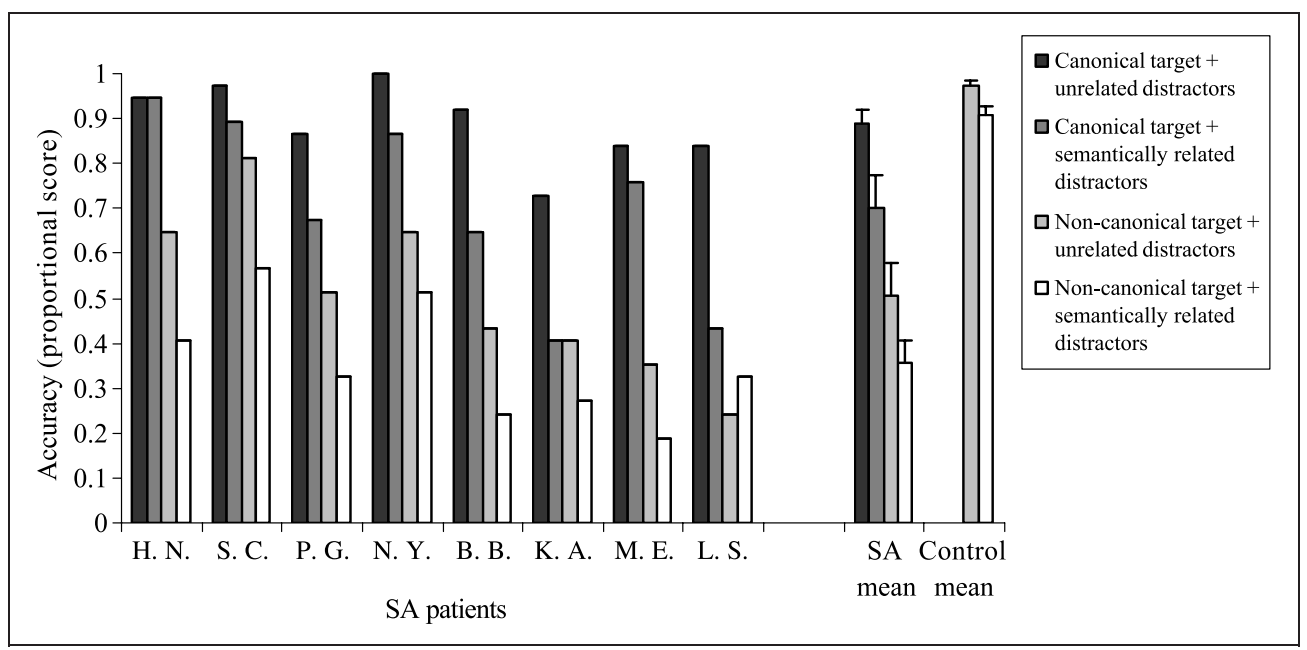


Figure 2. Object use under different levels of task constraint. Control participants assessed on the two most difficult conditions only (miming with/without a picture cue). Error bars show standard error of the mean.

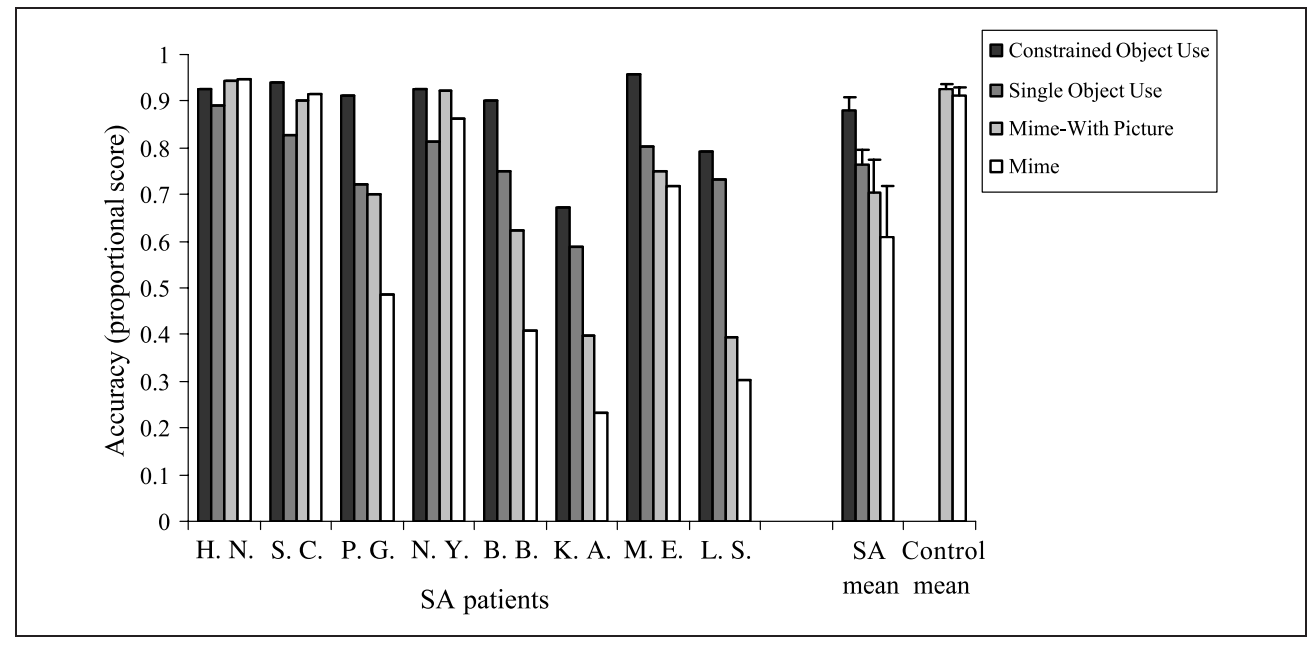

in these circumstances. As above, control participants were examined under the two most difficult task conditions (i.e., miming with and without a picture of the object).

\section{Results}

The patient data were examined using a repeated measures ANOVA including two factors: task type (mime vs. demonstration using real objects) and cue (no picture cue vs. picture present). There were main effects of task type [real object use $>$ mime, $F(1,7)=6.93, p=.03$ ] and picture cue [picture present $>$ no picture cue, $F(1,7)=22.14$, $p=.002]$. There was no interaction between the two factors $[F(1,7)>1$; see Figure 2$]$.

The control participants obtained good accuracy scores on the two hardest conditions of this task (miming: with picture 93\%; without picture cue 91\%). When these scores were compared with the SA groups' performance, we found main effects of cueing [with $>$ without picture, $F(1,14)=$ $9.74, p=.008$ ], group [controls $>$ SA patients, $F(1,14)=$ $8.30, p=.01]$, and a significant interaction between the two $[F(1,14)=5.14, p=.04]$. Using Bonferroni adjusted planned comparisons, the SA patients were found to obtain lower scores than the control participants in both miming tasks but the difference was greatest when no picture cue was provided [miming: with picture, $t(14)=2.77, p=.04$; without picture, $t(14)=2.90, p=.01]$.

\section{DISCUSSION}

This study examined the hypothesis that damage to a neural network encompassing left PFC and/or temporoparietal cortex leads to deregulated semantic cognition in some patients following a stroke; a condition known as SA. In particular, we explored the status of nonverbal conceptual knowledge in this condition, which has previously received far less attention than verbal processing even though damage to a core control component would be expected to affect all modalities together. In the present study, we systematically varied the degree and nature of control required in nonverbal tasks assessing knowledge of object use for the first time.

We found that SA patients' object use knowledge was dependent on the degree of control required by a particular task. In a six-alternative, forced-choice task, patients were able to select, with near ceiling accuracy, the object normally used to complete an everyday probe task (e.g., "kill a fly" $\rightarrow$ FLY SWAT). Accuracy decreased significantly, however, when semantic control was taxed in the following ways: (1) when the target response was not the object normally used to complete the probe task but constituted a noncanonical alternative (e.g., "kill a fly" $\rightarrow$ magazine) and (2) when highly distracting semantically related responses were embedded in the array of objects. These results closely parallel effects observed in word-based semantic association tasks (see Noonan et al., 2010; Jefferies \& Lambon Ralph, 2006). Because noncanonical objects would not be strongly associated with the probe task in everyday life, this condition is the most taxing for semantic control. In such circumstances, processes to select, retrieve, or bias the activation of object properties are required in order to determine which alternative item would be most suitable to use. Further, selection of the correct response was substantially more difficult when other closely associated concepts were presented alongside the correct response, suggesting that damage to the control component of semantic cognition made it more difficult for SA patients to overcome the effect of spreading activation and to inhibit these distracting items.

The dependence of object use on control demands was also demonstrated in the expressive domain. Patients found it more difficult to mime object use than demonstrate the same action with the object in hand. Miming was improved significantly when a picture of the target object was provided as well as its verbal label. Object use was most accurate when the actual object, a picture of its recipient, and a verbal description of the task were provided. By showing 
that it is possible to bolster object use with the provision of greater task constraints, these results are consistent with the view that semantic knowledge has not been lost in SA but a failure of regulatory control impairs the accurate use of knowledge in relatively unconstrained situations. These findings mirror SA patients' performance on picture naming tasks in which accuracy is enhanced significantly when naming is constrained by phonemic cues, as well as diminished with distracting miscues (Jefferies et al., 2008; Jefferies \& Lambon Ralph, 2006).

Our investigation builds on previous findings by uncovering the deregulated nature of semantic processing in SA for the first time in the nonverbal domain. In doing so, this study provides further support for the notion that semantic cognition is dependent on two principal components: (1) an amodal store of semantic representations, most likely underpinned by the ATL and (2) a regulatory control component supported by a neural network incorporating the left PFC and temporo-parietal region. Damage to either neural component can lead to similar overall levels of multimodal semantic impairment but more detailed inspection reveals qualitative differences reflective of the particular aspect of semantic cognition that has been affected (Jefferies \& Lambon Ralph, 2006). Although bilateral atrophy of the ATL causes progressive degradation of conceptual representations in SD, lesions affecting left $\mathrm{PFC}$ and/or temporo-parietal area result in a failure to control or shape semantic activation. As a consequence, relevant aspects of knowledge are not reliably brought to the fore. This means that SA patients are vulnerable to factors that load highly on semantic control processes such as weak/ nondominant associations and highly distracting foil responses. Further, it is possible to ameliorate the effects of deregulation temporarily by tightly constraining semantic activation through experimenter-controlled task conditions.

Consistent with our findings, neuroimaging studies commonly show that the left PFC and temporo-parietal region (i.e., the neural network damaged in SA) become more strongly activated during tasks that demand highly controlled use of conceptual knowledge (Badre \& Wagner, 2002; Wagner et al., 2001; Thompson-Schill et al., 1997; Demb et al., 1995). Moreover, frontal regions have been found to work collaboratively with posterior temporal and inferior parietal areas to control semantic processing (Nagel, Schumacher, Goebel, \& D’Esposito, 2008; Critescu, Devlin, \& Nobre, 2006; Rodd et al., 2005). In addition, the same neural network has been implicated in executive control beyond the verbal semantic domain (Rowe, Hughes, Eckstein, \& Owen, 2008; Peers et al., 2005). Strong connections between PFC and temporo-parietal area via the arcuate and superior longitudinal fasciculi (Parker et al., 2005; Gloor, 1997) reinforce the notion that these regions work closely together as a distributed functional system. The SA group assessed in this study displayed a mix of lesion profiles affecting either the left temporo-parietal region in isolation or in combination with PFC damage. Patients with lesions to one or both regions were behaviorally indistin- guishable in the present study; a finding that has been noted previously (Noonan et al., 2010; Jefferies \& Lambon Ralph, 2006; Berthier, 2001). For example, M. E. had damage restricted to posterior temporo-parietal regions with complete sparing of PFC but exhibited the same decline in performance with increasing control demands as cases with damage to both regions. Future studies should seek to recruit larger samples of anterior and posterior SA cases in order to explore whether there are more subtle differences between the two anatomical groups that the present case-series does not have sufficient power to detect. The emerging consensus that both prefrontal and temporoparietal regions work together to underpin cognitive control within different tasks and modalities fits well with our observation that damage to these two regions produces marked impairment of object use, as well as verbal semantic deficits, that are directly sensitive to manipulations of control demands.

\section{Acknowledgments}

We thank all of the patients and their carers for their continued support of our studies. We are also very grateful to Rachel Byrne, Linda Collier, Claire Slinger, and Mandy Galling for referring some of the patients to us, as well as Karen Sage for her help in classifying the patients' aphasic syndromes. Our thanks also go to Sheeba Ehsan for her help in collecting data and Krist Noonan for conducting the lesion analyses. This work was supported by an MRC-ESRC studentship (to F. Corbett), an RCUK fellowship (to E. Jefferies), and grants from NIMH (MH64445) and MRC (G0501632).

Reprint requests should be sent to Prof. Matthew A. Lambon Ralph, Neuroscience and Aphasia Research Unit (NARU), Zochonis Building, School of Psychological Sciences, University of Manchester, Oxford Road, Manchester, M13 9PL, UK, or via e-mail: matt.lambon-ralph@manchester.ac.uk.

\section{REFERENCES}

Badre, D., Poldrack, R. A., Pare-Blagoev, E. J., Insler, R. Z., \& Wagner, A. D. (2005). Dissociable controlled retrieval and generalized selection mechanisms in ventrolateral prefrontal cortex. Neuron, 47, 907-918.

Badre, D., \& Wagner, A. D. (2002). Semantic retrieval, mnemonic control, and prefrontal cortex. Behavioural and Cognitive Neuroscience Reviews, 1, 206-218.

Berthier, M. L. (2001). Unexpected brain-language relationships in aphasia: Evidence from transcortical sensory aphasia associated with frontal lobe lesions. Aphasiology, 15, 99-130.

Binney, R., Embleton, K. V., Jefferies, E., Parker, G. J. M., \& Lambon Ralph, M. A. (2010). The inferolateral aspects of the anterior temporal lobe are crucial in semantic memory: Evidence from a novel direct comparison of distortion-corrected fMRI, rTMS and semantic dementia. Cerebral Cortex, 20, 2728-2738.

Bozeat, S., Lambon Ralph, M. A., Patterson, K., Garrard, P., \& Hodges, J. R. (2000). Non-verbal semantic impairment in semantic dementia. Neuropsychologia, 38, 1207-1215.

Bozeat, S., Lambon Ralph, M. A., Patterson, K., \& Hodges, J. R. (2002). When objects lose their meaning: What happens to their use? Cognitive, Affective \& Behavioural Neuroscience, 2, 236-251. 
Burgess, P. W., \& Shallice, T. (1996). Bizarre responses, rule detection and frontal lobe lesions. Cortex, 32, 241-259.

Caramazza, A., \& Shelton, J. R. (1998). Domain-specific knowledge systems in the brain: The animate-inanimate distinction. Journal of Cognitive Neuroscience, 10, $1-34$.

Corbett, F., Jefferies, E., Ehsan, S., \& Lambon Ralph, M. A. (2009). Qualitatively different conceptual impairments in semantic dementia and semantic aphasia: Converging evidence from the non-verbal domain. Brain, 132, 2593-2608.

Critescu, T. C., Devlin, J. T., \& Nobre, A. C. (2006). Orientating attention to semantic categories. Neuroimage, 33, 1178-1187.

Damasio, H., \& Damasio, A. R. (1989). Lesion analysis in neuropsychology. New York: Oxford University Press.

Demb, J. B., Desmond, J. E., Wagner, A. D., Vaidya, C. J., Glover, G. H., \& Gabrieli, J. D. E. (1995). Semantic encoding and retrieval in the left inferior prefrontal cortex: A functional MRI study of task-difficulty and process specificity. Journal of Neuroscience, 15, 5870-5878.

Folstein, M. F., Folstein, S. E., \& McHugh, P. R. (1976). "Mini-mental state": A practical method for grading the mental state of patients for the clinician. Journal of Psychiatric Research, 12, 189-198.

Gibson, J. J. (1979). The ecological approach to visual perception. Boston: Houghton-Mifflin.

Gloor, P. (1997). The temporal lobe and the limbic system. Oxford: Oxford University Press.

Goldenberg, G. (1996). Defective imitation of gestures in patients with left and right hemisphere damage. Journal of Neurology, 61, 176-180.

Goodglass, H. (1983). The assessment of aphasia and related disorders (2nd ed.). Philadelphia, PA: Lea \& Febiger.

Grèzes, J., \& Decety, J. (2001). Does visual perception of object afford action? Evidence from a neuroimaging study. Neuropsychologia, 40, 212-222.

Howard, D., \& Patterson, K. (1992). Pyramid and palm trees: A test of semantic access from pictures and words. Bury St Edmunds, UK: Thames Valley Test Company.

Jefferies, E., Baker, S. S., Doran, M., \& Lambon Ralph, M. A. (2007). Refractory effects in stroke aphasia: A consequence of poor semantic control. Neuropsychologia, 45, 1065-1079.

Jefferies, E., \& Lambon Ralph, M. A. (2006). Semantic impairment in stroke aphasia versus semantic dementia: A case-series comparison. Brain, 129, 2132-2147.

Jefferies, E., Patterson, K., \& Lambon Ralph, M. A. (2008). Deficits of knowledge versus executive control in semantic cognition: Insights from cued naming. Neuropsychologia, 46, 649-658.

Kay, J., Lesser, R., \& Coltheart, M. (1992). Psycholinguistic assessments of language processing in aphasia (PALPA). Hove, UK: Erlbaum.

Koenig, K., \& Grossman, M. (2007). Process and content in semantic memory. In J. Hart \& M. A. Kraut (Eds.), Neural basis of semantic memory. Cambridge: Cambridge University Press.

Lambon Ralph, M. A., McClelland, J. L., Patterson, K., Galton, C. J., \& Hodges, J. R. (2001). No right to speak? The relationship between object naming and semantic impairment: Neuropsychological abstract evidence and a computational model. Journal of Cognitive Neuroscience, 13, 341-356.

Lambon Ralph, M. A., \& Patterson, K. (2008). Generalization and differentiation in semantic memory: Insights from semantic dementia. Year in Cognitive Neuroscience, 1124, 61-76.

Lambon Ralph, M. A., Sage, K., Jones, R. W., \& Mayberry, E. J. (2009). Coherent concepts are computed in the anterior temporal lobes. Proceedings of the National Academy of Sciences, U.S.A., 107, 2717-2722.

Milner, B. (1964). Effects of different brain lesions on card sorting: The role of the frontal lobes. Archives of Neurology, 9. $100-110$.

Mummery, C. J., Patterson, K., Price, C. J., Ashburner, J., Frackowiak, R. S. J., \& Hodges, J. R. (2000). A voxel-based morphometry study of semantic dementia: Relationship between temporal lobe atrophy and semantic memory. Annals of Neurology, 47, 36-45.

Nagel, I. E., Schumacher, E. H., Goebel, R., \& D’Esposito, M. (2008). Functional MRI investigation of verbal selection mechanisms in lateral prefrontal cortex. Neuroimage, 43, 801-807.

Nestor, P. J., Fryer, T. D., \& Hodges, J. R. (2006). Declarative memory impairments in Alzheimer's disease and semantic dementia. Neuroimage, 30, 1010-1020.

Noonan, K. A., Jefferies, E., Corbett, F., \& Lambon Ralph, M. A. (2010). Elucidating the nature of deregulated semantic cognition in semantic aphasia: Evidence for the roles of prefrontal and temporoparietal cortices, Journal of Cognitive Neuroscience, 22, 1597-1613.

Parker, G. J. M., Luzzi, S., Alexander, D. C., Wheeler-Kingshott, C. A. M., Clecarelli, O., \& Lambon Ralph, M. A. (2005). Lateralisation of ventral and dorsal auditory-language pathways in the human brain. Neuroimage, 24, 656-666.

Patterson, K., Nestor, P., \& Rogers, T. T. (2007). Where do you know what you know? The representation of semantic knowledge in the human brain. Nature Reviews Neuroscience, 8, 976-987.

Pavese, A., \& Buxbaum, L. J. (2002). Action matters: The role of action plans and object affordances in selection for action. Visual Cognition, 9, 559-590.

Peers, P. V., Ludwig, C. J. H., Rorden, C., Cusack, R., Bonfiglioli, C., Bundesen, C., et al. (2005). Attentional functions of parietal and frontal cortex. Cerebral Cortex, 15, 1469-1484

Raven, J. C. (1962). Coloured progressive matrices sets $A, A B, B$. London: H.K. Lewis.

Robertson, I. H., Ward, T., Ridgeway, V., \& Nimmo-Smith, I. (1994). The test of everyday attention. Flempton, UK: Thames Valley Test Company.

Rodd, J. M., Davis, M. H., \& Johnsrude, I. S. (2005). The neural mechanisms of speech comprehension: fMRI studies of semantic ambiguity. Cerebral Cortex, 15, 1261-1269.

Rogers, T. T., Lambon Ralph, M. A., Garrard, P., Bozeat, S., McClelland, J. L., Hodges, J. R., et al. (2004). Structure and deterioration of semantic memory: A neuropsychological and computational investigation. Psychological Review, 111, 205-235.

Rowe, J., Hughes, L., Eckstein, D., \& Owen, A. M. (2008). Ruleselection and action-selection have a shared neuroanatomical basis in the human prefrontal and parietal cortex. Cerebral Cortex, 18, 2275-2285.

Stuss, D. T., Levine, B., Alexander, M. P., Hong, J., Palumbo, C., Hamer, L., et al. (2000). Wisconsin Card Sorting Test performance in patients with focal frontal and posterior brain damage: Effects of lesion location and test structure on separable cognitive processes. Neuropsychologia, 38, 388-402.

Thompson-Schill, S. L. (2003). Neuroimaging studies of semantic memory: Inferring "how" from "where". Neuropsychologia, 41, 280-292.

Thompson-Schill, S. L., D'Esposito, M., Aguirre, G. K., \& Farah, M. J. (1997). Role of left inferior prefrontal cortex in retrieval of semantic knowledge: A reevaluation. Proceedings of the National Academy of Sciences, U.S.A., 94, 14792-14797. 
Thompson-Schill, S. L., D'Esposito, M., \& Kan, I. P. (1999). Effects of repetition and competition on activity in left prefrontal cortex during word generation. Neuron, 23, 513-522.

Thompson-Schill, S. L., Swick, D., Farah, M. J., D'Esposito, M., Kan, I. P., \& Knight, R. T. (1998). Verb generation in patients with focal frontal lesions: A neuropsychological test of neuroimaging findings. Proceedings of the National Academy of Sciences, U.S.A., 95, 15855-15860.

Wagner, A. D., Pare-Blagoev, E. J., Clark, J., \& Poldrack, R. A.
(2001). Recovering meaning: Left prefrontal cortex guides controlled semantic retrieval. Neuron, 31, 329-338.

Warrington, E. K., \& James, M. (1991). The visual object and space perception battery. Bury St. Edmunds, UK: Thames Valley Test Company.

Warrington, E. K., \& Shallice, T. (1984). Category specific semantic impairments. Brain, 107, 829-854.

Wechsler, D. (1987). Wechsler Memory Scale-Revised (WMS-R). New York: Psychological Corporation. 